\title{
Massive Electrodynamics and Magnetic Monopoles.
}

\author{
Mark Israelit ${ }^{\dagger}$, \\ $\dagger$ Department of Physics, University of Konstanz, PF 5560 M678, D-78434 Konstanz, Germany. On \\ leave From: Department of Physics, University of Haifa, Oranim, Tivon 36006, Israel.
}

\begin{abstract}
Including torsion in the geometric framework of the Weyl-Dirac theory we build up an action integral, and obtain From it a gauge covariant (in the Weyl sense) general relativistic massive electrodynamics. Photons having an arbitrary mass, electric, and magnetic currents (Dirac's monopole) coexist within this theory. Assuming that the space-time is torsionless, taking the photons mass zero, and turning to the Einstein gauge we obtain Maxwell's electrodynamics. PACS numbers: 04.20.Cv, 14.80.Hv,
\end{abstract}

14.70.Bh

\section{Introduction}

Ever after Dirac put forward the idea of magnetic poles [1], there has been continued interest and discussion on this subject. It is not surprising that this interest increased soon after Dirac [2] succeeded in setting up a more general theory, which led to a dynamical justification of his famous quantization condition. From an interesting review article by Blagojević and Senjanović [3], which covers over 150 works dealing with monopoles during six decades, one can see that theoretically oriented papers are dealing mostly with quantum aspects of the phenomenon, while the problem of constructing a satisfactory classical general relativistic framework was ignored in almost all works .

However, the problem must be considered also From the classical standpoint. First of all in order to quantize the fields it is necessary to have an action integral that provides us with a non-contradictory classical theory. Secondly, there are of course classical aspects of the monopole phenomenon. An example might be the explanation of magnetic fields of celestial bodies. Finally, if a magnetic charge (monopole) really exists, then Maxwell's electrodynamics that suffers From an asymmetry, regarding to electric, and magnetic currents, must be replaced by a generalized theory having a non-vanishing dual field tensor. Very interesting attempts in this direction were made by Hammond 四, who tried to describe electric and magnetic fields by torsion.

Another interesting problem is the photons mass. The massless photon became a tacit axiom of physics own to the success of quantum electrodynamics in predicting experiments with enormously high exactness. But the same results would be obtained with photons having mass $m_{\gamma}<10^{-48} \mathrm{~g}$ (cf. [5]). Moreover, as recently noted Ignatiev and Joshi [6, in some respects massive QED is simpler theoretically than the standard theory, and From the experimental point of view massive QED is as perfect as standard QED.

The purpose of this paper is to present a generalized eletrodynamics allowing both, electric and magnetic currents, and also massive photons. The theory is based on a generalization of the Weyl-Dirac geometry [7], [8]. The equations are derived From a geometrically justified action integral, and in the limiting case one has the ordinary Einstein-Maxwell theory.

\section{The Geometric Basis}

In order to develop an appropriate geometric basis we shall generalize the geometry of the Weyl-Dirac theory [7], [8 by including a contorsion term in the connection. We assume that there is a symmetric metric tensor $g_{\mu \nu}$, and an assymetric connection $\Gamma_{\mu \nu}^{\lambda}$ in each point of the manifold. The connection

\footnotetext{
${ }^{1}$ e-mail: Marc.Israelit@uni-konstanz.de , and: israelit@physics.technion.ac.il , (permanent e-address)
} 
will be split into three parts, the Christoffel symbol, the non-metricity, and the contorsion. With the non-metricity having the Weylian form one can write (cf. [9])

$$
\Gamma_{\mu \nu}^{\lambda}=\left\{\begin{array}{l}
\lambda \\
\mu \nu
\end{array}\right\}+g_{\mu \nu} w^{\lambda}-\delta_{\mu}^{\lambda} w_{\nu}-\delta_{\nu}^{\lambda} w_{\mu}+C_{\mu \nu}^{\lambda},
$$

where $w_{\mu}$ is the Weyl connection vector, and with the contorsion given in terms of the torsion tensor as follows

$$
C_{\mu \nu}^{\lambda}=\Gamma_{[\mu \nu]}^{\lambda}-g^{\lambda \beta} g_{\sigma \mu} \Gamma_{[\beta \nu]}^{\sigma}-g^{\lambda \beta} g_{\sigma \nu} \Gamma_{[\beta \mu]}^{\sigma},
$$

Actually one has now a Weyl geometry [7] with torsion, so that both, the direction and the length of a vector may change in the process of parallel displacement. For a vector $B^{\mu}$, having the length $B$, these changes are given by

$$
d B^{\mu}=-B^{\sigma} \Gamma_{\sigma \nu}^{\mu} d x^{\nu},
$$

and

$$
d B=B w_{\nu} d x^{\nu} .
$$

It follows that, after a parallel displacement around a parallelogram formed by $d x^{\mu}$ and $\delta x^{\nu}$, the length changes by

$$
\Delta B=-B W_{\mu \nu} d x^{\mu} \delta x^{\nu}
$$

with

$$
W_{\mu \nu}=w_{\mu, \nu}-w_{\nu, \mu} .
$$

This nonintegrability of length leads to an arbitrary standard of length, or gauge, at each point and hence to local gauge transformations

$$
B \rightarrow \bar{B}=e^{\lambda} B ; \quad g_{\mu \nu} \rightarrow \bar{g}_{\mu \nu}=e^{2 \lambda} g_{\mu \nu} ; \quad w_{\mu} \rightarrow \bar{w}_{\mu}=w_{\mu}+\lambda_{, \mu} .
$$

with $\lambda\left(x^{\mu}\right)$ being an arbitrary function. The third relation in (7) is the familiar transformation that one has in the Maxwell theory for the electromagnetic potential vector, and it led Weyl to identify $w_{\mu}$ with this vector, so that one has a connection between electromagnetism and geometry. In our generalization of the Weyl geometry we will assume that the torsion tensor is gauge invariant, so that in addition to (7) we have

$$
\Gamma_{[\mu \nu]}^{\lambda} \rightarrow \bar{\Gamma}_{[\mu \nu]}^{\lambda}=\Gamma_{[\mu \nu]}^{\lambda} .
$$

Considering a parallel displacement around an infinitesimal parallelogram, one can derive the curvature tensor

$$
K_{\mu \nu \sigma}^{\lambda}=-\Gamma_{\mu \nu, \sigma}^{\lambda}+\Gamma_{\mu \sigma, \nu}^{\lambda}-\Gamma_{\mu \nu}^{\alpha} \Gamma_{\alpha \sigma}^{\lambda}+\Gamma_{\mu \sigma}^{\alpha} \Gamma_{\alpha \nu}^{\lambda} .
$$

The two curvature tensors (6), and (9) may be used to build up an action integral. In order to get an action integral, which is invariant under both, the coordinate transformations, and the gauge transformations (7), and which agrees with the Einstein general relativity, Dirac [8] introduced a scalar field $\beta\left(x^{\mu}\right)$. It is assumed that under a gauge transformation (7) the gauging function $\beta\left(x^{\mu}\right)$ changes according to

$$
\beta \rightarrow \bar{\beta}=e^{-\lambda} \beta .
$$

For the torsionless case Dirac built up a geometrically based action. He made use of the curvature scalar $K=g^{\mu \nu} K_{\mu \nu \lambda}^{\lambda}$ formed From (9), of a term $W_{\mu \nu} W^{\mu \nu}$ constructed From the Weylian length curvature tensor (6), as well as of the vector $w_{\mu}$ and the scalar $\beta$. This action has the following form [8]

$$
I_{\text {Dirac }}=\int\left[W^{\mu \nu} W_{\mu \nu}-\beta^{2} K+k\left(\beta_{, \underline{\mu}}+\beta w^{\mu}\right)\left(\beta_{, \mu}+\beta w_{\mu}\right)+2 \Lambda \beta^{4}+L_{\text {matter }}\right](-g)^{1 / 2} d x
$$

where $\Lambda$ is the cosmological constant, $k$ an arbitrary parameter, $L_{\text {matter }}$ is the Lagrangian density of matter, and an underlined index is to be raised with $g^{\mu \nu}$. In order to get From (11) the Maxwell field equations Dirac took $k=6$. Later Rosen [10] gave a detailed discussion on the Weyl-Dirac theory. He pointed out that for $k \neq 6$, one obtains a Proca equation 15 instead of Maxwell's one.For $k-6<0$ From the standpoint of quantum mechanics the Proca field may be interpreted as an ensemble of bosons, particles of spin 1 and finite mass. These massive vector bosons, named weylons, were used for obtaining non-baryonic dark matter in the universe [11], 12. 
In the present work it will be assumed that electromagnetism may be described by the Proca equations, so that the particles will be regarded rather as massive photons. Moreover, we incorporate torsion into the space, so that the connection (2), as well as the curvature (9) contain torsion. For a moment let us consider the second derivative of a given vector $B_{\mu}$, with respect to the connection $\Gamma_{\mu \nu}^{\lambda}$ defined in (11). (cf. [9]):

$$
B_{\mu: \nu: \sigma}-B_{\mu: \sigma: \nu}=B_{\lambda} K_{\mu \nu \sigma}^{\lambda}-2 B_{\mu: \alpha} \Gamma_{[\nu \sigma]}^{\alpha} .
$$

In the last term of (12) appear additional geometric properties, that are not expressed neither in (6), nor in (9). In order to include them in the Lagrangian one has to replace $B_{\mu}$ by a fundamental geometric quantity that is already present in the framework. For this purpose let us take the tensor $W_{\mu \nu}$ (It may be recalled here that $g_{\mu \nu: \lambda}=-2 g_{\mu \nu} w_{\lambda}$, so that $W_{\mu \nu}$ may be regarded as the second derivative of $\left.g_{\mu \nu}\right)$. By this choice the torsional curvature term takes on the form

$$
a W_{\mu \lambda: \alpha} \Gamma_{[\nu \sigma]}^{\alpha}
$$

with $a$ being an abitrary constant.

In the next section we will make use of (6), (9), and (13) for building up an action .

\section{Variational Principle}

Let us start from Dirac's action (11). It is convenient to express the curvature scalar $K$ explicitly in terms of the Christoffelsymbol $\left\{\begin{array}{l}\lambda \\ \mu \nu\end{array}\right\}$, the Weyl connection vector $w_{\mu}$, and the torsion tensor $\Gamma_{[\mu \nu]}^{\lambda}$ according to (1), and (2). We will include also the scalar $a W_{\mu \nu ; \alpha} \Gamma_{[\lambda \sigma]}^{\alpha} g^{\mu \lambda} g^{\nu \sigma}$, stemming from (12), but with $\Gamma$ differentiation (:) replaced by the Riemannian one (;) (formed with $\left\{\begin{array}{l}\lambda_{\mu \nu} \\ \text { \} }\end{array}\right.$ ). Thus we have the following action integral:

$$
\begin{array}{r}
I=\int\left(W^{\mu \nu} W_{\mu \nu}-\beta^{2} R\right. \\
+\beta^{2}(k-6) w_{\mu} w^{\mu}+2(k-6) \beta w^{\mu} \beta_{, \mu}+k \beta_{, \mu} \beta_{, \underline{\mu}}+8 \beta \Gamma_{[\lambda \alpha]}^{\alpha} \beta_{, \underline{\lambda}} \\
+\beta^{2}\left(2 \Gamma_{[\mu \lambda]}^{\alpha} \Gamma_{[\underline{\mu} \alpha]}^{\lambda}-\Gamma_{[\sigma \alpha]}^{\alpha} \Gamma_{[\underline{\omega} \omega]}^{\omega}+\Gamma_{[\mu \lambda]}^{\alpha} \Gamma_{[\underline{\mu} \underline{\lambda}]}^{\omega} g_{\alpha \omega}+8 \Gamma_{[\sigma \alpha]}^{\alpha} w^{\sigma}\right) \\
\left.+a W_{\mu \nu ; \alpha} \Gamma_{[\underline{\mu} \underline{\mu}]}^{\alpha}+2 \Lambda \beta^{4}+L_{\text {matter }}\right)(-g)^{1 / 2} d x
\end{array}
$$

with $R$ being the Riemannian curvature scalar.

One can readily prove (From the field equations) that $a=4$. Below this value will be taken. Varying in (14) $w_{\mu}$ one obtains the following field equation

$$
\left(W^{\mu \nu}-2 \Gamma_{[\underline{\mu} \underline{\underline{ }}] ; \alpha}^{\alpha}\right)_{; \nu}=(1 / 2) \beta^{2}(k-6) W^{\mu}+2 \beta^{2} \Gamma_{[\underline{\mu} \alpha]}^{\alpha}+4 \pi J^{\mu}
$$

where $W_{\mu}$ stands for the gauge-invariant Weyl vector (cf. (ఫ))

$$
W_{\mu}=w_{\mu}+(\ln \beta)_{, \mu}
$$

and

$$
16 \pi J^{\mu}=\frac{\delta L_{\text {matter }}}{\delta w_{\mu}}
$$

Considering the variation of (14) with respect to $\Gamma_{[\mu \nu]}^{\lambda}$ we obtain a second field equation :

$$
\begin{array}{r}
W_{; \lambda}^{\mu \nu}=\beta^{2}\left(\delta_{\lambda}^{\mu} W^{\nu}-\delta_{\lambda}^{\nu} W^{\mu}\right)+\beta^{2}\left(\delta_{\lambda}^{\nu} g^{\mu \sigma}-\delta_{\lambda}^{\mu} g^{\nu \sigma}\right) \Gamma_{[\sigma \alpha]}^{\alpha} \\
+(1 / 2) \beta^{2}\left(g^{\sigma \nu} \delta_{\omega}^{\mu} \delta_{\lambda}^{\rho}-g_{\lambda \omega} g^{\sigma \mu} g^{\rho \nu}-g^{\sigma \mu} \delta_{\omega}^{\nu} \delta_{\lambda}^{\rho}\right) \Gamma_{[\sigma \rho]}^{\omega}-4 \pi \Omega_{\lambda}^{[\mu \nu]}
\end{array}
$$

where

$$
16 \pi \Omega_{\lambda}^{[\mu \nu]}=\frac{\delta L_{\text {matter }}}{\delta \Gamma_{[\mu \nu]}^{\lambda}}
$$


The form of eq. (15) justifies introducing the field

$$
\Phi_{\mu \nu}=W_{\mu \nu}-2 \Gamma_{[\mu \nu] ; \alpha}^{\alpha} \equiv W_{\mu ; \nu}-W_{\nu ; \mu}-2 \Gamma_{[\mu \nu] ; \alpha}^{\alpha} .
$$

If one varies in (14) the metric tensor $g^{\mu \nu}$, one obtains the following equation for the gravitational field:

$$
\begin{aligned}
\beta^{2} G^{\mu \nu} & =-8 \pi T^{\mu \nu}-8 \pi\left(\widetilde{M}^{\mu \nu}-\bar{M}^{\mu \nu}\right)+2 \beta\left(g^{\mu \nu} \beta_{; \alpha ; \underline{\alpha}}-\beta_{; \underline{\mu} ; \underline{\nu}}\right)+4 \beta_{, \underline{\mu}} \beta_{, \underline{\nu}}-g^{\mu \nu} \beta_{, \underline{\alpha}} \beta_{, \alpha} \\
& +(k-6) \beta^{2}\left(W^{\mu} W^{\nu}-1 / 2 g^{\mu \nu} W^{\sigma} W_{\sigma}\right)+4 \beta^{2} \Gamma_{[\sigma \alpha]}^{\alpha}\left(g^{\sigma \nu} W^{\mu}+g^{\sigma \mu} W^{\nu}-g^{\mu \nu} W^{\sigma}\right) \\
& +\beta^{2} \Gamma_{[\sigma \tau]}^{\alpha} \Gamma_{[\lambda \rho]}^{\omega} f(g ; \delta)-g^{\mu \nu} \beta^{4} \Lambda,
\end{aligned}
$$

with

$$
\begin{array}{r}
f(g ; \delta)=2 \delta_{\alpha}^{\tau} \delta_{\omega}^{\rho}\left(g^{\lambda \sigma} g^{\mu \nu}-g^{\lambda \mu} g^{\sigma \nu}-g^{\lambda \nu} g^{\sigma \mu}\right)+\delta_{\omega}^{\tau} \delta_{\alpha}^{\rho}\left(g^{\lambda \mu} g^{\sigma \nu}+g^{\lambda \nu} g^{\sigma \mu}-g^{\lambda \sigma} g^{\mu \nu}\right) \\
+g^{\tau \rho}\left(2 g^{\lambda \mu} g^{\sigma \nu} g_{\alpha \omega}-\delta_{\omega}^{\mu} \delta_{\alpha}^{\nu} g^{\lambda \sigma}-\frac{1}{2} g^{\mu \nu} g^{\lambda \sigma} g_{\alpha \omega}\right),
\end{array}
$$

and where $8 \pi T^{\mu \nu}=\delta L_{\text {matter }} / \delta g_{\mu \nu}$. The modified energy density tensors of the field are defined as follows

$$
4 \pi \widetilde{M}^{\mu \nu}=(1 / 4) g^{\mu \nu} \Phi^{\alpha \beta} \Phi_{\alpha \beta}-\Phi^{\mu \alpha} \Phi_{\alpha}^{\nu}
$$

and

$$
4 \pi \bar{M}^{\mu \nu}=(1 / 4) g^{\mu \nu}\left(\Phi^{\alpha \beta}-W^{\alpha \beta}\right)\left(\Phi_{\alpha \beta}-W_{\alpha \beta}\right)-\left(\Phi^{\mu \alpha}-W^{\mu \alpha}\right)\left(\Phi_{\alpha}^{\nu}-W_{\alpha}^{\nu}\right)
$$

Contracting eq. (21) we get:

$$
\begin{aligned}
& R-\left(8 \pi / \beta^{2}\right) T+(6 / \beta) \beta_{; \alpha ; \underline{\alpha}}-(k-6) W^{\sigma} W_{\sigma}-8 \Gamma_{[\sigma \alpha]}^{\alpha} W^{\sigma} \\
& +\quad 4 \Gamma_{[\lambda \alpha]}^{\alpha} \Gamma_{[\underline{\lambda} \beta]}^{\beta}-2 \Gamma_{[\lambda \omega]}^{\rho} \Gamma_{[\underline{\lambda} \rho]}^{\omega}-g_{\alpha \omega} \Gamma_{[\lambda \sigma]}^{\alpha} \Gamma_{[\underline{\lambda \sigma}]}^{\omega}-4 \beta^{2} \Lambda=0 .
\end{aligned}
$$

Finally varying in (14) the gauging function $\beta$, one has

$$
\begin{aligned}
& \beta R+k \beta \beta_{; \alpha ; \underline{\alpha}}=\beta\left(8 \Gamma_{[\lambda \alpha]}^{\alpha} w^{\lambda}-4 \Gamma_{[\lambda \alpha] ; \lambda}^{\alpha}-4 \Gamma_{[\lambda \alpha]}^{\alpha} \Gamma_{[\underline{\lambda} \beta]}^{\beta}+2 \Gamma_{[\lambda \rho]}^{\alpha} \Gamma_{[\underline{\lambda} \alpha]}^{\rho}+g^{\mu \nu} g_{\alpha \omega} \Gamma_{[\lambda \mu]}^{\alpha} \Gamma_{[\underline{\lambda} \nu]}^{\omega}\right) \\
+ & \beta(k-6)\left(w^{\sigma} w_{\sigma}-w_{; \sigma}^{\sigma}\right)+4 \beta^{3} \Lambda+8 \pi B,
\end{aligned}
$$

with

$$
16 \pi B=\frac{\delta L_{\text {matter }}}{\delta \beta} .
$$

From equations (24), and (25) one obtains the following relation

$$
(k-6)\left(\beta^{2} W^{\sigma}\right)_{; \sigma}+8 \pi(T-\beta B)+4\left(\beta^{2} \Gamma_{[\underline{\sigma} \alpha]}^{\alpha}\right)_{; \sigma}=0 .
$$

Now, let us go back to the field equations (15) - (20).Contracting (18) one has

$$
W_{; \nu}^{\mu \nu}=-3 \beta^{2} W^{\mu}+2 \beta^{2} \Gamma_{[\underline{\mu} \nu]}^{\nu}-4 \pi \Omega_{\nu}^{[\mu \nu]} .
$$

From (15) and (28) one has two interesting conservation laws

$$
(k-6)\left(\beta^{2} W^{\mu}\right)_{; \mu}+8 \pi J_{; \mu}^{\mu}+4\left(\beta^{2} \Gamma_{[\underline{\mu \nu}]}^{\nu}\right)_{; \mu}=0
$$

and

$$
3\left(\beta^{2} W^{\mu}\right)_{; \mu}+4 \pi \Omega_{\nu ; \mu}^{[\mu \nu]}-2\left(\beta^{2} \Gamma_{[\underline{\mu \nu}]}^{\nu}\right)_{; \mu}=0
$$

For the scalar $\int L_{\text {matter }}(-g)^{1 / 2} d^{4} x$ one can consider an infinitesimal transformation of coordinates. This leads to the following conservation law :

$$
T_{\mu ; \sigma}^{\sigma}+J_{; \sigma}^{\sigma} w_{\mu}+J^{\sigma} W_{\mu \sigma}-B \beta_{; \mu}-\Omega_{\lambda}^{[\sigma \rho]} \Gamma_{[\sigma \rho] ; \mu}^{\lambda}+\left(2 \Omega_{\lambda}^{[\rho \sigma]} \Gamma_{[\rho \mu]}^{\lambda}-\Omega_{\mu}^{[\rho \lambda]} \Gamma_{[\rho \lambda]}^{\sigma}\right)_{; \sigma}=0
$$


Considering for the same integral an infinitesimal gauge transformation,one obtains the relation

$$
J_{; \mu}^{\mu}=T-\beta B
$$

One readily sees that $(32)$ is compatible with relations (27), and (29).

In order to get a non-vanishing magnetic current we will use the well-known procedure (cf. e. g. [13], [14])of building up dual field equations. With (20) we can rewrite (15) as follows

$$
\Phi_{; \nu}^{\mu \nu}=(1 / 2) \beta^{2}(k-6) W^{\mu}+2 \beta^{2} \Gamma_{[\underline{\mu} \alpha]}^{\alpha}+4 \pi J^{\mu} .
$$

Further, comparing between (15) and (28) we obtain

$$
2 \Gamma_{[\underline{\mu} \underline{\underline{\nu}}] ; \alpha ; \nu}^{\alpha}=-(1 / 2) k \beta^{2} W^{\mu}-4 \pi\left(J^{\mu}+\Omega_{\nu}^{[\mu \nu]}\right) .
$$

We can generalize expression (34) writing :

$$
2 \Gamma_{[\underline{\mu} \underline{\underline{\nu}}] ; \alpha ; \lambda}^{\alpha}=-(k / 6) \beta^{2}\left(\delta_{\lambda}^{\nu} W^{\mu}-\delta_{\lambda}^{\mu} W^{\nu}\right)-(4 \pi / 3)\left(J^{\mu} \delta_{\lambda}^{\nu}-J^{\nu} \delta_{\lambda}^{\mu}\right)-4 \pi \Omega_{\lambda}^{[\mu \nu]} .
$$

Making use of (20), and (35) we can write the cyclic permutation

$$
\Phi_{\mu \nu ; \lambda}+\Phi_{\lambda \mu ; \nu}+\Phi_{\nu \lambda ; \mu}=4 \pi\left(\Omega_{\mu[\nu \lambda]}+\Omega_{\lambda[\mu \nu]}+\Omega_{\nu[\lambda \mu]}\right)=4 \pi \Theta_{\mu \nu \lambda},
$$

where $\Omega_{\lambda[\mu \nu]} \equiv g_{\mu \alpha} g_{\nu \beta} \Omega_{\lambda}^{[\alpha \beta]}$.

Now, let us define the dual field

$$
\widetilde{\Phi}^{\mu \nu}=-\frac{1}{2(-g)^{1 / 2}} \varepsilon^{\mu \nu \alpha \beta} \Phi_{\alpha \beta}
$$

and the current

$$
L^{\sigma}=-\frac{1}{6(-g)^{1 / 2}} \varepsilon^{\sigma \mu \nu \lambda} \Theta_{\mu \nu \lambda} .
$$

where $\varepsilon^{\mu \nu \alpha \beta}$ stands for the completely antisymmetric Levi-Civita symbol, and $\varepsilon^{0123}=1$. ( A book by O. Veblen [16], containing useful information on Levi-Civita symbols, must be mentioned here.) For the dual of eq. (33) we can now write:

$$
\widetilde{\Phi}_{; \nu}^{\mu \nu}=4 \pi L^{\mu}
$$

Finally, From (39) one obtains

$$
L_{; \mu}^{\mu}=0
$$

that may be interpreted as a conservation law for the magnetic current density.

Equations (33), and (39) may be intepreted as describing an electromagnetic field induced by the electric current density $J^{\mu}$ and by the magnetic current density $L^{\mu}$. These equations are covariant under gauge transformations (7), (10). It is worth noting that eq. (33) is rather a Proca equation then a Maxwell one. Turning to the Einstein gauge $\beta=1$, considering a torsionless geometry $\Gamma_{[\mu \nu]}^{\lambda}=0$, (equation (36), (38), and (39) vanish identically in that case), and taking $k=6$ we obtain From (21), and (33) the Einstein-Maxwell equations and From eq. (31) we obtain the energy conservation law in its conventional form.

\section{A Possible Theory}

In the previous section a general formalism was developed. In the present section we will specify it. Let us choose for the torsion tensor a representation suitable for describing magnetism. We recall that in our case the Weyl connection vector $w_{\mu}$ acts as the electromagnetic potential vector. In order to describe the magnetic field and to invoke a nonzero magnetic current density we need another vector. The torsion can be broken in three irreducible parts (cf. e.g. [1] , 19]): a trace part, a traceless one, and a totally antisymmetric part. Let us asumme that the first two parts vanish. In this case the totally 
antisymmetric torsion tensor $\Gamma_{[\mu \nu]}^{\lambda}$ may be represented by a vector.If we introduce the auxiliary torsion tensors:

$$
\Gamma_{\lambda[\mu \nu]}=g_{\sigma \lambda} \Gamma_{[\mu \nu]}^{\sigma} ; \quad \Gamma^{\lambda[\mu \nu]}=g^{\alpha \mu} g^{\beta \nu} \Gamma_{[\alpha \beta]}^{\lambda} .
$$

we can express the torsion by means of a vector $V^{\mu}$ as follows

$$
\Gamma_{\lambda[\mu n u]}=(-g)^{1 / 2} \varepsilon_{\lambda \mu \nu \sigma} V^{\sigma} ; \quad \Gamma^{\lambda[\mu \nu]}=-(-g)^{-1 / 2} \varepsilon^{\lambda \mu \nu \sigma} V_{\sigma}
$$

This leads to

$$
\Gamma_{[\mu \nu]}^{\nu}=0 .
$$

From (42) one can also derive the following simple formula:

$$
\Gamma_{; \alpha}^{\alpha[\mu \nu]}=\frac{\varepsilon^{\mu \nu \alpha \sigma}}{2(-g)^{1 / 2}}\left(V_{\alpha ; \sigma}-V_{\sigma ; \alpha}\right) .
$$

Making use of (44) we can rewrite the following exact expressions for the field tensors, defined by (20), and (37)

$$
\Phi^{\mu \nu}=\left(W_{; \underline{\nu}}^{\mu}-W_{; \underline{\mu}}^{\nu}\right)-\frac{\varepsilon^{\mu \nu \alpha \sigma}}{(-g)^{1 / 2}}\left(V_{\alpha ; \sigma}-V_{\sigma ; \alpha}\right)
$$

and

$$
\widetilde{\Phi}^{\mu \nu}=-2\left(V_{; \underline{\nu}}^{\mu}-V_{; \underline{\mu}}^{\nu}\right)-\frac{\varepsilon^{\mu \nu \alpha \sigma}}{2(-g)^{1 / 2}}\left(W_{\alpha ; \sigma}-W_{\sigma ; \alpha}\right) .
$$

Inserting these into equations (33) and (39), and making use of (43), we obtain

$$
W_{; \underline{i} ; \nu}^{\mu}-W_{; \underline{\mu} ; \nu}^{\nu}-\frac{\varepsilon^{\mu \nu \alpha \sigma}}{(-g)^{1 / 2}}\left(V_{\alpha, \sigma, \nu}-V_{\sigma, \alpha, \nu}\right)=(1 / 2)(k-6) \beta^{2} W^{\mu}+4 \pi J^{\mu}
$$

and

$$
-2\left(V_{; \underline{\nu} ; \nu}^{\mu}-V_{; \underline{\mu} ; \nu}^{\nu}\right)-\frac{\varepsilon^{\mu \nu \alpha \sigma}}{2(-g)^{1 / 2}}\left(W_{\alpha, \sigma, \nu}-W_{\sigma, \alpha, \nu}\right)=4 \pi L^{\mu} .
$$

One can readily see that in (47), and in (48) the termswith the Levi-Civita symbols vanish identically, so that one is left with the following field equations

$$
\Phi_{; \nu}^{\mu \nu}=W_{; \underline{i} ; \nu}^{\mu}-W_{; \underline{\mu} ; \nu}^{\nu}=(1 / 2)(k-6) \beta^{2} W^{\mu}+4 \pi J^{\mu}
$$

and

$$
\widetilde{\Phi}_{; \nu}^{\mu \nu}=\left(V_{; \underline{\nu} ; \nu}^{\mu}-V_{; \underline{\mu} ; \nu}^{\nu}\right)=-2 \pi L^{\mu}
$$

From (49) one sees that

$$
\Phi_{; \nu}^{\mu \nu}=W_{; \nu}^{\mu \nu} .
$$

Thus, comparing (49) with (28), and taking into account (43), and (51) one obtains

$$
\Omega_{\nu}^{[\mu \nu]}=J^{\mu}+(1 / 8 \pi) \beta^{2} k W^{\mu} .
$$

Now let us consider the tensor $\Omega_{\lambda}^{[\mu \nu]}$ as defined by (19). It has to satisfy condition (52), and its structure must be in accordance with (42). It is convenient to introduce auxiliary tensors

$$
\Omega^{\lambda[\mu \nu]}=g^{\lambda \sigma} \Omega_{\sigma}^{[\mu \nu]} ; \quad \Omega_{\lambda[\mu \nu]}=g_{\alpha \mu} g_{\beta \nu} \Omega_{\lambda}^{[\alpha \beta]} .
$$

An appropriate choice is

$$
\Omega_{\lambda[\mu \nu]}=(1 / 3)\left(g_{\nu \lambda} J_{\mu}-g_{\mu \lambda} J_{\nu}\right)+\frac{k \beta^{2}}{24 \pi}\left(g_{\nu \lambda} W_{\mu}-g_{\mu \lambda} W_{\nu}\right)+(-g)^{1 / 2} \varepsilon_{\lambda \mu \nu \sigma} l^{\sigma},
$$

and

$$
\Omega^{\lambda[\mu \nu]}=(1 / 3)\left(g^{\nu \lambda} J^{\mu}-g^{\mu \lambda} J^{\nu}\right)+\frac{k \beta^{2}}{24 \pi}\left(g^{\nu \lambda} W^{\mu}-g^{\mu \lambda} W^{\nu}\right)-(-g)^{-1 / 2} \varepsilon^{\lambda \mu \nu \sigma} l_{\sigma} \quad ;
$$


with $l_{\mu}$ being a gauge invariant vector.

Making use of (41) - (43) one finds that equation (21) now takes on the form

$$
\begin{array}{r}
G^{\mu \nu}=-\left(8 \pi / \beta^{2}\right) T^{\mu \nu}-\left(8 \pi / \beta^{2}\right)\left(\widetilde{M}^{\mu \nu}-\bar{M}^{\mu \nu}\right)+(2 / \beta)\left(g^{\mu \nu} \beta_{; \underline{\alpha} ; \alpha}-\beta_{; \underline{j} ; \underline{\nu}}\right) \\
+\left(1 / \beta^{2}\right)\left(4 \beta_{; \underline{\mu}} \beta_{; \underline{\nu}}-g^{\mu \nu} \beta_{; \underline{\alpha}} \beta_{; \alpha}\right)+(k-6)\left(W^{\mu} W^{\nu}-\frac{1}{2} g^{\mu \nu} W^{\sigma} W_{\sigma}\right)-g^{\mu \nu} V^{\sigma} V_{\sigma}-2 V^{\mu} V^{\nu} .
\end{array}
$$

The equations describing the electromagnetic fields are given by (49), (50), and for the magnetic current density vector $L^{\mu}$ one has by (36), (38), and (54)

$$
L^{\sigma}=3 l^{\sigma}
$$

From (49), (50) one can also derive a current conservation law

$$
(k-6)\left(\beta^{2} W^{\mu}\right)_{; \mu}+8 \pi J_{; \mu}^{\mu}=0,
$$

as well the conservation law for the magnetic currrent (49).

It is remarkable that in (49), (50) the electric currentdensity vector $J^{\mu}$ appears as a creator of the $W$-part of the field, while the magnetic current density vector $L^{\mu}$ creates the $V$-part.

\section{The Einstein Gauge}

The torsionless Weyl-Dirac theory with $k=6$ takes on the form of the Einstein-Maxwell theory if one choose the Einstein gauge $\beta=1$. (cf. [8], , 10], [17]). Thus the Eistein gauge may help to understanding the theory.

In this section we consider the theory, possessing torsion, and allowing arbitrary values for $k$, which was developed in the previous section. Turning to the Einstein gauge, we set

$$
\beta=1 .
$$

By condition (59) we also have $w^{\mu}$ instead $W^{\mu}$, andequation (56) now takes on the form

$$
\begin{array}{r}
G^{\mu \nu}=-8 \pi T^{\mu \nu}-8 \pi\left(\widetilde{M}^{\mu \nu}-\bar{M}^{\mu \nu}\right)-(k-6)\left(w^{\mu} w^{\nu}-(1 / 2) g^{\mu \nu} w^{\sigma} w_{\sigma}\right) \\
-2 V^{\mu} V^{\nu}-g^{\mu \nu} V^{\sigma} V_{\sigma} .
\end{array}
$$

The energy conservation law can be obtained from (31) by complicated and very lengthy calculations. Alternatively one can, making use of the Bianchi identities, and taking the divergence, derive this law from equation (60).

Let us choose the second way. We have $G_{\mu ; \nu}^{\nu}=0$, so that(60) leads to

$$
8 \pi\left(T_{\mu ; \nu}^{\nu}+\widetilde{M}_{\mu ; \nu}^{\nu}-\bar{M}_{\mu ; \nu}^{\nu}\right)+(k-6)\left(w_{\mu} w^{\nu}-(1 / 2) \delta_{\mu}^{\nu} w_{\sigma} w^{\sigma}\right)_{; \nu}+2\left(V_{\mu} V^{\nu}\right)_{; \nu}+\left(V_{\sigma} V^{\sigma}\right)_{; \mu}=0 .
$$

Making in (61) use of definitions (22), and (23), and of equations (36), (49), (51), (54), (57) one obtains

$$
\begin{array}{r}
8 \pi\left(T_{\mu ; \nu}^{\nu}+\Psi_{\mu \sigma} J^{\sigma}\right)+4 \pi \sqrt{-g} \varepsilon_{\alpha \beta \mu \sigma} W^{\alpha \beta} L^{\sigma}+(k-6)\left(\Psi_{\mu \sigma}+W_{\mu \sigma}\right) w^{\sigma} \\
+(k-6) w_{\mu} w_{; \nu}^{\nu}+2 V^{\nu}\left(V_{\mu ; \nu}+V_{\nu ; \mu}\right)+2 V_{\mu} V_{; \nu}^{\nu}=0 .
\end{array}
$$

For a moment let us go back to (49). With a new parameter $\kappa^{2} \equiv(1 / 2)(6-k)$ we can rewrite this equation as

$$
\Phi_{; \nu}^{\mu \nu}=w_{; \underline{\nu} ; \nu}^{\mu}-w_{; \underline{\mu} ; \nu}^{\nu}=-\kappa^{2} w^{\mu}+4 \pi J^{\mu}
$$

In absent of electric currents in a certain region we obtain From (58), and (59)

$$
w_{; \nu}^{\nu}=0
$$

so that equation (63) may be rewritten in the following form

$$
w_{; \underline{\nu} ; \nu}^{\mu}+w^{\nu} R_{\nu}^{\mu}+\kappa^{2} w^{\mu}=0 \quad ;
$$


with $R_{\nu}^{\mu}$ being the Ricci tensor, formed from the usual Christoffel symbols. If the curvature in the current-free region is negligible, one obtains the Proca [15] equation for the vector field $w^{\mu}$

$$
w_{; \underline{\nu} ; \nu}^{\mu}+\kappa^{2} w^{\mu}=0
$$

From the quantum mechanical standpoint this equation describes a particle having spin 1 and mass that in conventional units is given by

$$
m=(\hbar / c) \kappa=(\hbar / c) \sqrt{\frac{6-k}{2}},
$$

thus, for $k<6$ one obtains massive field particles, photons.

In the special case when, $V^{\mu}=0$, and $k=6$, one obtains From (50) $L^{\mu}=0$, so that equations (60), and (63) turn into the equations of the Einstein-Maxwell theory, while (62) becomes the usual energy conservation law.

\section{Discussion}

The Weyl geometry [7] is doubtless the most aesthetic generalization of the Riemannian geometry, the last being the framework of general relativity. Dirac [8] modified the Weyl theory. In order to build upan action integral, which is coordinate invariant, and gauge invariant,and which agrees with the general relativity theory, Dirac introduced a scalar gauging function, $\beta$. The modified Weyl-Dirac theoryoffers a complete basis for deriving gravitation, and electromagnetism From geometry (cf. e.g. [17], [18]).

Generalizing the Weyl-Dirac framework [7], 8], [10], we have developed in the present work a geometrically based general relativistic theory, possessing intrinsic electric andmagnetic currents and admitting massive photons. Torsion is includedinto the geometry, so that the linear connection (cf.(1)) is made now of three parts, the metric (Christoffel) term, the Weylian non-metricity, and contorsion. Following Dirac [8] we also make use of the gauging function.

The general procedure is given in section 3. Varying in the actionthe metric tensor $g_{\mu \nu}$, the Weyl connection vector $w_{\mu}$, the torsion tensor $\Gamma_{[\mu \nu]}^{\lambda}$, and the gauge function $\beta$ one obtains the equations, as well as conservation laws. Making use of these equations one can introduce such a field tensor (cf. (20) ) that its dual (37) defines a nonzero magnetic current density vector. The eletromagnetic field is related to the electric current by a Proca eq. (cf. (33) ) instead of a Maxwell one, so that massive photons are expected. However one can choose the value of an arbitrary parameter to get massless photons and Maxwell equations. The whole theory is gauge covariant in the Weyl sense.

In section 4 we specify the torsion tensor, assuming that it has a totally antisymmetric structure. Making this assumption enables to represent the torsion as a dual of a vector field. This representationresults in a simple form of the field equations. One obtains also charge conservation laws (40), (58). From the field equation (49) one sees that the electromagnetic potential vector $W_{\mu}$ has two sources, the electric current density vector $J_{\mu}$, and a Proca term, while according to (50) the magnetic vector $V_{\mu}$ is created only by magnetic currents $L_{\mu}$. The two field vectors are coupled by the energy conservation law and by the equation for the gravitation field (56) (cf. definitions (22), (23)).

Assuming $\beta=1$ one turns to the Einstein gauge, in which the field, and its dual are given by (63), and (50) respectively. The coupling of these two fields and their currents is manifested in the energy conservation law (62). So one sees that the magnetic current vector $L^{\mu}$ is coupled to the electricfield $W^{\mu \nu}$, while the electric current $J^{\mu}$ is coupledto the whole field. The mass term (with the factor $(k-6)$ ) is alsoconnected to both fields. One also obtains an equation (66) that may be treated as describing massive field particles, photonsin current-free regions. The photon mass is fixed by an arbitrary parameter $\kappa$ that may be chosen to give any extremely small value for $m_{\gamma}$. Choosing a vanishing photon mass we obtain From (63) the classical Maxwell equation, possessingthe usual Maxwell gauge invariance.

A geometric based theory is proposed. It is shown that electric, and intrinsic magnetic currents, as well massive photons coexist within this framework. If one drops the torsion, and sets $k=6$ one has the Weyl-Dirac theory, which in the Einstein gauge takes on the form of Einstein-Maxwell. 


\section{Acknowledgments}

The author takes this opportunity to express his cordial thanks to Professor HEINZ DEHNEN for very interesting discussions.

\section{References}

[1] P. A. M. Dirac, Proc. Roy. Soc. A133, 60, (1931).

[2] P. A. M. Dirac, Phys. Rev. 74, 817, (1948).

[3] M. Blagojević, and P. Senjanović, Physics Reports, 157, 234, (1988).

[4] R. T. Hammond, Il Nuovo Cimento 108 B, 725, (1993).

[5] A. S. Golhaber, and M. M. Nieto, Rev. Mod. Phys. 43, 277, (1971).

[6] A. Yu. Ignatiev, and G. C. Joshi, Phys. Rev. D. 53, 984, (1996).

[7] H. Weyl, Ann. Phys. (Leipzig) 59, 101, (1919).

[8] P. A. M. Dirac, Proc. Roy. Soc. A333, 403, (1973).

[9] J. Schouten, Ricci Calculus,(Springer Verlag, Berlin, 1954).

[10] N. Rosen, Found. Phys. 12, 213, (1982).

[11] M. Israelit and N. Rosen, Found. Phys. 22, 555, (1992).

[12] M. Israelit and N. Rosen, Found. Phys. 24, 901, (1994).

[13] B. Felsager, Geometry, Particles and Fields, (Odense University Press 1981).

[14] L. D. Landau and E. M. Lifshitz, The Classical Theory of Fields, (Pergamon, Oxford, 1975).

[15] A. L. Proca, J. Phys. Rad. 7, 347, (1936)

[16] O. Veblen, Invariants of Quadratic Differential Forms , ( University Press, Cambridge, 1962).

[17] M. Israelit and N. Rosen, Found. Phys. 13, 1023, (1983).

[18] M. Israelit, Found. Phys. 19, 35, (1989)

[19] K. Hayashi and T. Shirafuji, Prog. Theor. Phys., 64, 866, (1980). 